\title{
Lattice QCD approach to nuclear force
}

\author{
N. Ishii* \\ Center for Computational Sciences, University of Tsukuba, Tsukuba 305-8577, Japan \\ E-mail: ishii@ribf.riken.jp
}

\section{S. Aoki}

RIKEN BNL Research Center, Brookhaven National Laboratory, Upton, New York 11973, USA

Graduate School of Pure and Applied Sciences, University of Tsukuba, Tsukuba 305-8571,

Japan

E-mail: saoki@het.ph.tsukuba.ac.jp

\section{T. Hatsuda}

Department of Physics, University of Tokyo, Tokyo 113-0033, Japan

E-mail: hatsuda@phys.s.u-tokyo.ac.jp

\begin{abstract}
We present our updated results of the nucleon-nucleon potential in quenched lattice QCD simulations with the plaquette gauge action and the Wilson quark action on the $32^{4}\left(\simeq(4.4 \mathrm{fm})^{4}\right)$ lattice. From the equal-time Bethe-Salpeter (BS) wave function, the NN potential is constructed through the Schrödinger-type equation. Resulting NN potential has all the qualitative features which phenomenological potentials commonly have: the repulsive core at short distance and the attractive well at medium and long distances. In the $L \rightarrow \infty$ limit, our NN potential is guaranteed to reproduce the scattering length obtained from the Lüscher's formula. The quark mass dependence of the NN potential is studied with $m_{\pi} \sim 380,529,731 \mathrm{MeV}$. The results suggest that both the repulsive core at short distance and the attractive well at medium distance are enhanced in the light quark mass region.
\end{abstract}

The XXV International Symposium on Lattice Field Theory

July 30-4 August 2007

Regensburg, Germany

\footnotetext{
*Speaker.
} 


\section{Introduction}

The nuclear force is the essential ingredient in nuclear physics. Since H. Yukawa has introduced the pion 72 yeas ago [1], enormous efforts have been devoted to understand the origin of the nuclear force [2]. At medium to long distances $(r \gtrsim 1.2 \mathrm{fm})$, the nuclear force is attractive, which is essential for the existence of bound nuclei. Furthermore, the nuclear force exhibits the repulsive core at short distance $(r \lesssim 0.7 \mathrm{fm})$, which is intimately related to the stability of heavy nuclei and neutron stars. The field theoretical treatment on the basis of the pion and heavy meson exchanges has reasonable success at long and medium distances. However, the repulsive core at short distance, which was first introduced by R. Jastrow 56 years ago to explain the high energy behavior of the NN phase shifts [3], has not been understood with firm theoretical ground. Since the nucleons begin to overlap at short distances, the quark and gluon structure of the nucleon is expected to play an important role. In this respect, the first principle lattice QCD calculation is desired to serve as the most powerful tool to attack the problems of the nuclear force. There exits a previous attempt to apply the formalism of the static $q \bar{q}$ potential to NN potential [4]. However, the method which employs the static quarks is not faithful to the scattering data of nucleons.

We have recently generalized the method developed by CP-PACS collaboration [5] for the $\pi \pi$ scattering length and have carried out a first calculation of the NN potential from lattice QCD [6]. In our method, the Bethe-Salpeter wave function, whose asymptotic form leads to correct NN phase shift in the asymptotic regime, is introduced to construct the NN potential through the Schrödinger-type equation. The resulting NN potential has all the qualitative features required by phenomenology, i.e., the repulsive core at short distance and the attractive well at medium to long distances. In the report, we extend our previous calculations by increasing statistics and by introducing different quark masses corresponding to the pion masses, $m_{\pi} \simeq 380,529,731 \mathrm{MeV}$. The results show that both the repulsive core and attractive well are enhanced in the light quark mass region.

\section{The formalism}

We begin with the Schrödinger-type equation, which is satisfied by the Bethe-Salpeter wave function for the NN system,

$$
\begin{aligned}
\left(\vec{\nabla}^{2}-k^{2}\right) \Psi_{E}(\vec{r}) & =m_{N} \sum_{\vec{r}^{\prime}} U\left(\vec{r}, \vec{r}^{\prime}\right) \Psi_{E}\left(\vec{r}^{\prime}\right) \\
\Psi_{E, \alpha \beta}(\vec{r}) & \equiv \lim _{t \rightarrow+0}\left\langle 0\left|T\left[p_{\alpha}(\vec{x}, t) n_{\beta}(\vec{y}, 0)\right]\right| N N(E)\right\rangle
\end{aligned}
$$

where $\vec{r} \equiv \vec{x}-\vec{y} \cdot p_{\alpha}(x)$ and $n_{\beta}(y)$ denote the standard local interpolating fields for nucleons,

$$
p_{\alpha}(x) \equiv \varepsilon_{a b c}\left(u_{a}^{T}(x) C \gamma_{5} d_{b}(x)\right) u_{c, \alpha}(x), \quad n_{\beta}(y) \equiv \varepsilon_{a b c}\left(u_{a}^{T}(y) C \gamma_{5} d_{b}(y)\right) d_{c, \beta}(y),
$$

where $a, b$ and $c$ denote the color indices. $\alpha$ and $\beta$ denote the Dirac indices. $C$ denotes the charge conjugation matrix. (For derivation of Eq. (2.1), see Refs. [5, 7, 8].) $U\left(\vec{r}, \vec{r}^{\prime}\right)$, which does not depend on $E$, plays a role of the non-local interaction kernel [8]. The most general (off-shell) form of $\mathrm{NN}$ potential after imposing constraints arising from various symmetries is analyzed in Ref. [9]. 
By applying the derivative expansion up to $O\left(\vec{\nabla}^{2}\right)$, we obtain

$$
\begin{aligned}
U\left(\vec{r}, \vec{r}^{\prime}\right) & =U_{1}\left(\vec{r}, \vec{r}^{\prime}\right)+\left(\vec{\tau}_{1} \cdot \vec{\tau}_{2}\right) U_{\tau \tau}\left(\vec{r}, \vec{r}^{\prime}\right) \\
& =\mathscr{P}^{(I=0)} U^{(I=0)}\left(\vec{r}, \vec{r}^{\prime}\right)+\mathscr{P}^{(I=1)} U^{(I=1)}\left(\vec{r}, \vec{r}^{\prime}\right) \\
U^{(I)}\left(\vec{r}, \vec{r}^{\prime}\right) & =V^{(I)} \cdot \delta\left(\vec{x}-\vec{x}^{\prime}\right) \\
V^{(I)} & =V_{0}^{(I)}+\left(\vec{\sigma}_{1} \cdot \vec{\sigma}_{2}\right) V_{\sigma}^{(I)}+S_{12} V_{\mathrm{T}}^{(I)}+(\vec{L} \cdot \vec{S}) V_{\mathrm{LS}}^{(I)}+\left\{\vec{\sigma}_{1} \cdot \vec{L}, \vec{\sigma}_{2} \cdot \vec{L}\right\} V_{\mathrm{LL}}^{(I)}+\left\{\vec{\sigma}_{1} \cdot \vec{\nabla}, \vec{\sigma}_{2} \cdot \vec{\nabla}\right\} V_{\mathrm{pp}}^{(I)} \\
& \simeq V_{0}^{(I)}(r)+\left(\vec{\sigma}_{1} \cdot \vec{\sigma}_{2}\right) V_{\sigma}^{(I)}(r)+S_{12} V_{\mathrm{T}}^{(I)}(r)+(\vec{L} \cdot \vec{S}) V_{\mathrm{LS}}^{(I)}(r)+O\left(\vec{\nabla}^{2}\right),
\end{aligned}
$$

where $\mathscr{P}^{(I=0)} \equiv\left(1-\vec{\tau}_{1} \cdot \vec{\tau}_{2}\right) / 4$ and $\mathscr{P}^{(I=1)} \equiv\left(3+\vec{\tau}_{1} \cdot \vec{\tau}_{2}\right) / 4$ denote the projection matrices to $I=0$ and $I=1$ subspaces, respectively. $\{*, *\}$ denotes the anti-commutator. $S_{12} \equiv\left(\vec{\sigma}_{1} \cdot \vec{r}\right)\left(\vec{\sigma}_{2} \cdot \vec{r}\right) / r^{2}-$ $\vec{\sigma}_{1} \cdot \vec{\sigma}_{2}, \vec{L} \equiv-i \vec{r} \times \vec{\nabla}$ and $\vec{S} \equiv\left(\vec{\sigma}_{1}+\vec{\sigma}_{2}\right) / 2 . V_{0}^{(I)}, V_{\sigma}^{(I)}, V_{\mathrm{T}}^{(I)}, V_{L L}^{(I)}$ and $V_{\mathrm{pp}}^{(I)}$ are functions of $\vec{r}^{2}, \vec{\nabla}^{2}$, and $\vec{L}^{2}$. We combine the 1st and the 2nd terms in the last line as $V_{\mathrm{C}}^{(I)}(r) \equiv V_{0}^{(I)}(r)+\left(\vec{\sigma}_{1} \cdot \vec{\sigma}_{2}\right) V_{\sigma}^{(I)}(r)$, and refer to $V_{\mathrm{C}}^{(I)}(r)$ as the central force. $V_{\mathrm{T}}^{(I)}(r)$ and $V_{\mathrm{LS}}^{(I)}(r)$ are refereed to as the tensor force and the $L S$ force, respectively. These three forces play major roles in conventional nuclear physics.

In $\mathrm{QCD}$, the closest concept to the quantum mechanical wave function is provided by the equal-time Bethe-Salpeter (BS) wave function Eq. (2.2). Note that Eq. (2.2) represents a probability amplitude to find three quarks at $\vec{x}$ and another three quarks at $\vec{y}$. It is possible to show that Eq. (2.2) has a proper asymptotic behavior at $|\vec{x}-\vec{y}| \rightarrow \infty[8]$. For example, in the ${ }^{1} S_{0}$ channel, we have

$$
\Psi_{E}(\vec{r}) \rightarrow e^{i \delta_{0}(k)} \frac{\sin \left(k r+\delta_{0}(k)\right)}{k r} .
$$

The BS wave function is obtained from the large $t$ behavior of the "four-point" correlator of the nucleon

$$
\left\langle 0\left|T\left[p(\vec{x}, t) n(\vec{x}, t) W\left(t=t_{0}\right)\right]\right| 0\right\rangle=\sum_{n}\langle 0|p(\vec{x}, 0) n(\vec{y}, 0)| n\rangle e^{-i E_{n}\left(t-t_{0}\right)}\langle n|W(t=0)| 0\rangle .
$$

Here, $W(t) \equiv \bar{P}(t) \bar{N}(t)$ represents the wall source, where $P(t)$ and $N(t)$ are defined as Eq. (2.3) with the quark fields $q(\vec{x}, t)$ replaced by $Q(t) \equiv \sum_{\vec{x}} q(\vec{x}, t)$. Since contributions from all excited states are exponentially suppressed in the large $t$ region, we are left with the BS wave function for the ground state. In this report, we consider only ${ }^{1} S_{0}$ and ${ }^{3} S_{1}$ channels whose BS wave functions are

$$
\begin{aligned}
& \Psi\left(\vec{r} ;{ }^{1} S_{0}\right)=\frac{1}{24} \sum_{R \in O} \frac{1}{L^{3}} \sum_{\vec{X}}\left(\sigma_{2}\right)_{\alpha \beta}\left\langle 0\left|p_{\alpha}(R \cdot \vec{r}+\vec{X}) n_{\beta}(\vec{X})\right| N N(E)\right\rangle \\
& \Psi\left(\vec{r} ;{ }^{3} S_{1}\right)=\frac{1}{24} \sum_{R \in O} \frac{1}{L^{3}} \sum_{\vec{X}}\left(\sigma_{2} \sigma_{3}\right)_{\alpha \beta}\left\langle 0\left|p_{\alpha}(R \cdot \vec{r}+\vec{X}) n_{\beta}(\vec{X})\right| N N(E)\right\rangle,
\end{aligned}
$$

where the summations over $R \in O$ are performed for cubic transformation group. The summations for $\vec{X}$ are performed to select the zero total spatial momenta.

First we consider the Schrödinger equation in ${ }^{1} S_{0}$ channel. Owing to the identical nature of the nucleons, two nucleon system in ${ }^{1} S_{0}$ channel is iso-vector. Since the contributions from the tensor force and the LS force vanish in this channel, we are left with the following Schrödinger equation,

$$
\left(-\frac{1}{2 \mu} \vec{\nabla}^{2}+V_{\mathrm{C}}^{(I=1)}(r)\right) \Psi\left(\vec{r}^{1}{ }^{1} S_{0}\right)=\frac{k^{2}}{2 \mu} \Psi\left(\vec{r}^{1}{ }^{1} S_{0}\right)
$$




\begin{tabular}{cccccll}
\hline$\kappa$ & $N_{\text {conf }}$ & $m_{\pi}[\mathrm{MeV}]$ & $m_{\mathrm{N}}[\mathrm{MeV}]$ & $t-t_{0}$ & $E\left({ }^{1} S_{0}\right)[\mathrm{MeV}]$ & $E\left({ }^{3} S_{1}\right)[\mathrm{MeV}]$ \\
\hline 0.1640 & 1000 & $732.1(4)$ & $1558.4(63)$ & 7 & $-0.400(83)$ & $-0.480(97)$ \\
0.1665 & 2000 & $529.0(4)$ & $1333.8(82)$ & 6 & $-0.509(94)$ & $-0.560(114)$ \\
0.1678 & 2021 & $379.7(9)$ & $1196.6(32)$ & 5 & $-0.675(264)$ & $-0.968(374)$ \\
\hline
\end{tabular}

Table 1: The number of gauge configurations $N_{\text {conf }}$, the pion mass $m_{\pi}$, the nucleon mass $m_{\mathrm{N}}$, time-slice $t-t_{0}$ on which BS wave functions are measured, and the non-relativistic energies $E \equiv k^{2} /(2 \mu)$ for ${ }^{1} S_{0}$ and ${ }^{3} S_{1}$ channels.

where $\mu \equiv m_{N} / 2$ denotes the reduced mass of the nucleon. $k$ plays the role of the "asymptotic momentum". Since $V_{\mathrm{C}}^{(I=1)}(r)=V_{0}^{(I=1)}(r)-3 V_{\sigma}^{(I=1)}(r)$ is an ordinary function, which does not involve a derivative nor matrix structure, we arrange the Schrödinger equation to obtain

$$
V_{\mathrm{C}}^{(I=1)}(r)=\frac{k^{2}}{2 \mu}+\frac{1}{2 \mu} \frac{\vec{\nabla}^{2} \Psi\left(\vec{r},{ }^{1} S_{0}\right)}{\Psi\left(\vec{r},{ }^{1} S_{0}\right)}
$$

Next, we consider the Schrödinger equation in ${ }^{3} S_{1}$ channel. In this case, two nucleon system is iso-scalar. Unlike ${ }^{1} S_{0}$ case, this channel receives a non-vanishing contribution from the tensor force, which provides a coupling to ${ }^{3} D_{1}$ channel. ${ }^{3} D_{1}$ channel receives a contribution from the LS force. In this way, we have three unknowns, i.e., $V_{\mathrm{C}}^{(I=0)}(r), V_{\mathrm{T}}^{(I=0)}(r)$ and $V_{\mathrm{LS}}^{(I=0)}(r)$ with two equations, i.e., ${ }^{3} S_{1}$ and ${ }^{3} D_{1}$. $\left(V_{\mathrm{C}}^{(I=0)}(r)=V_{0}^{(I=0)}(r)+V_{\sigma}^{(I=0)}(r)\right.$ should not be confused with $V_{\mathrm{C}}^{(I=1)}(r)$.) To obtain these three forces exactly, we need one more equation such as the Schrödinger equation in ${ }^{3} D_{2}$ channel. In this report, we do not pursue this direction. Instead, we adopt the same procedure as the ${ }^{1} S_{0}$ channel. This leads to the so-called "effective central force" $V_{\mathrm{C}}^{\text {eff }}(r)$ which takes into account the ${ }^{3} D_{1}$ channel indirectly through the tensor force.

\section{The lattice $Q C D$ result}

We employ the standard plaquette action on $32^{4}$ lattice with $\beta=5.7$ to generate quenched gauge configurations. The gauge configurations are picked up every 200 sweeps after skipping 3000 sweeps for thermalization. Quark propagators are generated by employing the standard Wilson quark action with $\kappa=0.1640,0.1665$ and 0.1678 . The scale unit $1 / a=1.44(2) \mathrm{GeV}$ $(a \simeq 0.137 \mathrm{fm})$ is introduced from the rho meson mass in the chiral limit [10]. The physical size of our lattice corresponds to $L \sim 4.4 \mathrm{fm}$. The number of gauge configurations $N_{\text {conf }}$, the pion mass $m_{\pi}$, the nucleon mass $m_{\mathrm{N}}$ are summarized in Table 1. (For $\kappa=0.1678,24$ gauge configurations are identified as exceptional configurations, which are not used in the calculations.)

The periodic (Dirichlet) boundary condition is imposed on the quark fields along the spatial (temporal) direction. We adopt the wall source on the time-slice $t=t_{0} \equiv 5$. The BS wave functions are measured on the time-slice $t-t_{0}=7,6,5$ for $\kappa=0.1640,0.1646,0.1678$, respectively. The ground state saturation is examined by t-dependence of the $\mathrm{NN}$ potential. We employ the nearest neighbor representation of the discretized Laplacian as $\vec{\nabla}^{2} f(\vec{x}) \equiv \sum_{i=1}^{3}\left\{f\left(\vec{x}+a \vec{e}_{i}\right)+f\left(\vec{x}-a \vec{e}_{i}\right)\right\}-$ $6 f(\vec{x})$, where $\vec{e}_{i}$ denotes the unit vector along the $i$-th coordinate axis. BS wave functions are fully measured for $|\vec{r}| \lesssim 0.7 \mathrm{fm}$, where rapid changes of BS wave function and NN potential are expected. 

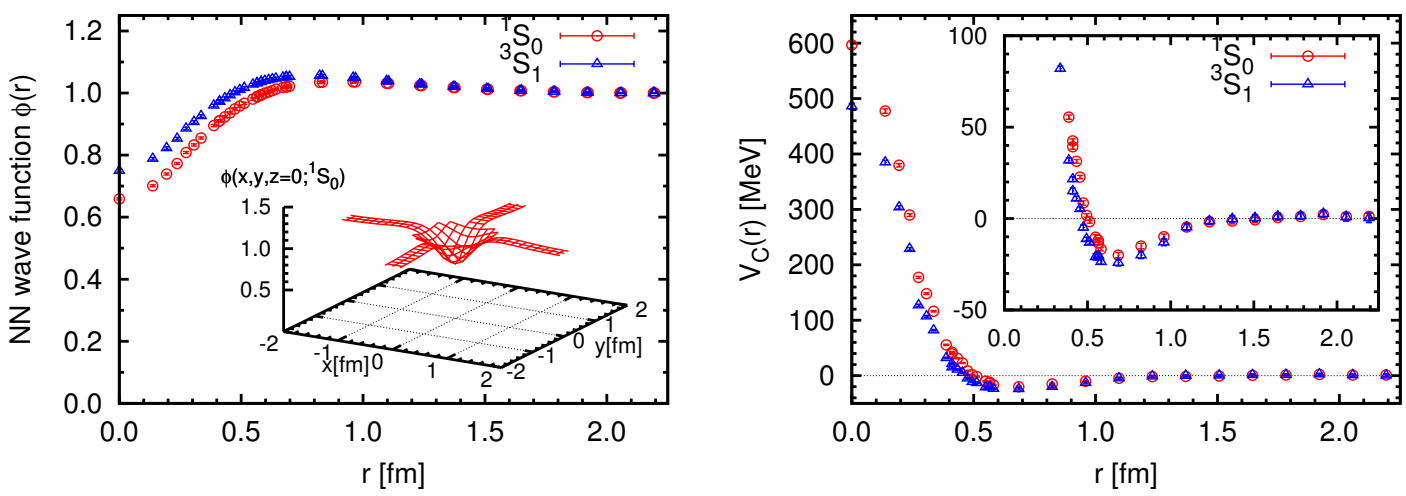

Figure 1: NN wave functions in ${ }^{1} S_{0}$ and ${ }^{3} S_{1}$ channels (left), and NN potentials (right), i.e., central force in ${ }^{1} S_{0}$ channel and effective central force in ${ }^{3} S_{1}$ channel for $\kappa=0.1665$. The inset of the left figure is a 3D plot of the wave function $\phi\left(x, y, z=0 ;{ }^{1} S_{0}\right)$.

Since the changes are rather modest for $|\vec{r}| \gtrsim 0.7 \mathrm{fm}$, the measurement of BS wave functions is restricted on the coordinate axes and their nearest neighbors to reduce the calculational cost. The "asymptotic momentum" $k^{2}$ is obtained by fitting the BS wave function: We use the Green's function of the Helmholtz equation,

$$
G\left(\vec{r} ; k^{2}\right) \equiv \frac{1}{L^{3}} \sum_{\vec{n} \in \mathbb{Z}^{3}} \frac{\exp (i 2 \pi \vec{n} \cdot \vec{r} / L)}{(2 \pi / L)^{2} \vec{n}^{2}-k^{2}}
$$

as the fit function by regarding the overall numerical factor and $k^{2}$ as fit parameters. (An appropriate regularization is assumed in this representation of Green's function.) The fits are performed outside of the range of NN interaction, which is determined by examining $\vec{\nabla}^{2} \Psi(\vec{x}) / \Psi(\vec{x})$ [5].

Fig. 1 (left) shows the BS wave functions in ${ }^{1} S_{0}$ and ${ }^{3} S_{1}$ channels for $\kappa=0.1665$. The suppression of the wave function in the region $r \lesssim 0.5 \mathrm{fm}$ indicate the existence of repulsion at short distance. Fig. 1 (right) shows the reconstructed NN potentials for $\kappa=0.1665$, i.e., the central force for ${ }^{1} S_{0}$ channel and the effective central force for ${ }^{3} S_{1}$ channel. (See Table 1, for the values of the non-relativistic energies $E \equiv k^{2} /(2 \mu)$ in Eq. (2.10).) We see that our NN potentials have repulsive cores of $500-600 \mathrm{MeV}$ in the short distance region $(r \lesssim 0.5 \mathrm{fm})$ and attractions of about $30 \mathrm{MeV}$ in the medium distance region $(0.5 \lesssim r \lesssim 1.2 \mathrm{fm})$. Both the repulsive core at short distance and the attractive well at medium distance are weaker than those expected phenomenologically. This is due to the heavy quark mass in our simulations In the light quark mass region, pion can propagate longer distance, which is expected to enhance the attraction at medium and long distance.

To see the quark mass dependence in a quantitative manner, we show the NN potentials for three different quark masses in ${ }^{1} S_{0}$ channel in Fig. 2. As the quark mass decreases, the repulsive core at short distance is enhanced rapidly, whereas the attraction at medium distance is modestly enhanced. This indicates that it is important to perform the lattice QCD calculation in the lighter quark mass region in order to compare our result with experimental data.

Several comments are in order here.

(i) The net interaction is attractive even in the presence of the repulsive core. Indeed, Lüscher's finite volume method leads to the attractive scattering length, i.e., $a_{0}\left({ }^{1} S_{0}\right)=0.115(26), 0.126(25)$, 


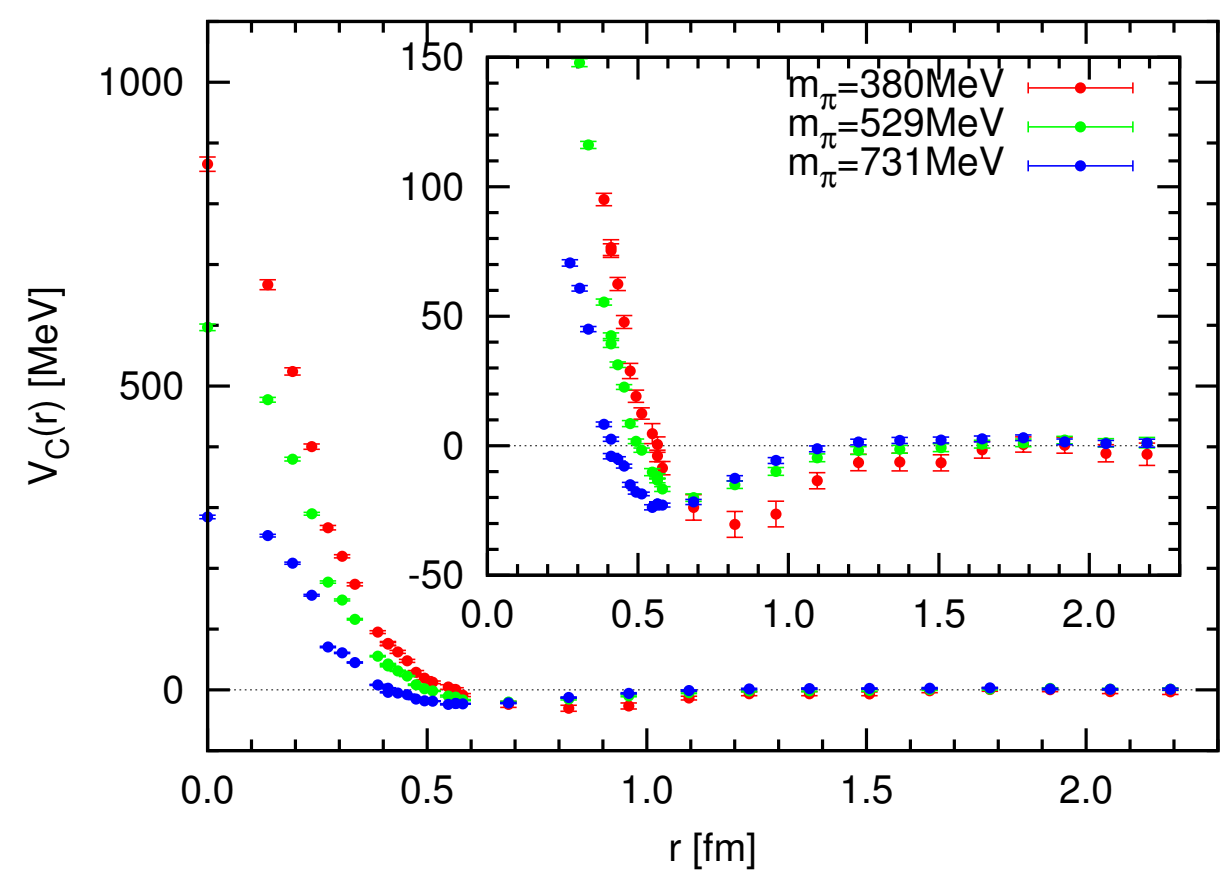

Figure 2: Central forces in ${ }^{1} S_{0}$ channel for three quark masses.

$0.159(66)$ fm and $a_{0}\left({ }^{3} S_{1}\right)=0.140(31), 0.140(31), 0.252(104)$ fm for $\kappa=0.1640,0.1665,0.1678$, respectively. The attractive nature of our potential is qualitatively understood by the Born approximation formula for the scattering length $a_{0} \simeq-m_{N} \int V_{\mathrm{C}}(r) r^{2} d r$. Owing to the volume factor $r^{2} d r$, the attraction at medium distance overcomes the repulsive core at short distance.

(ii) There is a considerable discrepancy between the above scattering lengths and the empirical values, i.e., $a_{0}^{(\exp )}\left({ }^{1} S_{0}\right) \sim 20 \mathrm{fm}$ and $a_{0}^{(\exp )}\left({ }^{3} S_{1}\right) \sim-5 \mathrm{fm}$. This is attributed to the heavy quark mass employed in our simulations. If we can get closer to the physical quark mass, there appears an "unitary region" where the NN scattering length becomes singular as a function of the quark mass and changes sign $[11,12]$. The singular point is related to the threshold of bound state formation. This is why the physical scattering length is positively large in the ${ }^{1} S_{0}$ channel (no bound state) and is negatively large in the ${ }^{3} S_{1}$ channel (deuteron bound state).

\section{Summary}

We have extended our previous results of the nuclear force on the lattice by increasing statistics and adopting different quark masses. The NN potentials in the ${ }^{1} S_{0}$ and ${ }^{3} S_{1}$ channels have all the qualitative features which phenomenological NN potentials commonly have, i.e., the repulsive core at short distance and attractive well at medium to long distances. The quark mass dependence of the NN potential shows that the repulsive core at short distance is enhanced rapidly, and the attraction at medium distance is modestly enhanced. These results suggest that, in order to compare our results with the experimental data, it is important to perform the lattice QCD Monte Carlo calculation in the lighter quark mass region. 
Although the BS wave functions are proved to have the universal behavior as Eq. (2.6) at large distance, its short distance behavior is afflicted with the operator dependence. We can avoid this subtlety by resorting to the inverse scattering theory, which guarantees the unique existence of energy-independent, local, hermitian potential. Studies along this line together with the NN potential measured in different $E$ and the derivation of the tensor force will be reported elsewhere [8].

\section{Acknowledgements}

Lattice QCD Monte Carlo calculation has been done with IBM Blue Gene/L at KEK. This research was partly supported by the Ministry of Education, Science, Sports and Culture, Grant-inAid (Nos. 13135204, 15540251, 15540254, 18540253, 19540261).

\section{References}

[1] H. Yukawa, Proc. Phys. Math. Soc. Japan, 17, 48 (1935).

[2] R. Machleidt and I. Slaus, J. Phys. G27, R69 (2001).

[3] R. Jastrow, Phys. Rev. 81, 165 (1951).

[4] T.T. Takahashi, T. Doi and H. Suganuma, AIP Conf. Proc. 842, 249 (2006).

[5] S. Aoki et al., CP-PACS Collab., Phys. Rev. D71, 094504 (2005).

[6] N. Ishii, S. Aoki and T. Hatsuda, Phys. Rev. Lett. 99, 022001 (2007).

[7] C.J.D. Lin, G. Martinelli, C.T. Sachrajda and M. Testa, Nucl. Phys. B619, 467 (2001).

[8] S. Aoki, T. Hatsuda and N. Ishii, in preparation.

[9] S. Okubo and R.E. Marshak, Ann. Phys. 4, 166 (1958).

[10] M. Fukugita, Y. Kuramashi, M. Okawa, H. Mino and A. Ukawa, Phys. Rev. D52, 3003 (1995).

[11] Y. Kuramashi, Prog. Theor. Phys. Suppl. 122, 153 (1996).

[12] S.R. Beane, P.F. Bedaque, K. Orginos and M.J. Savage, Phys. Rev. Lett. 97, 012001 (2006). 